\title{
POSSIBLE APPLICATIONS OF FOCUSED IMPEDANCE METHOD (FIM) IN BIOMEDICAL AND OTHER AREAS OF STUDY
}

\author{
K Siddique-e Rabbani and M Abdul Kadir \\ Department of Biomedical Physics \& Technology \\ University of Dhaka, Dhaka, Bangladesh \\ email: rabbani@univdhaka.edu,kadir@univdhaka.edu
}

\begin{abstract}
Focused Impedance Method (FIM) based on 8, 6 and 4 electrode configurations have been innovated and developed for measuring the electrical impedance of a localised zone in a volume conductor, all based on tetrapolar impedance technique. The zone localization in FIM has been visualized through equipotential distributions, current density at the target point and perturbation produced at the potential measuring electrodes. The predicted behaviour has been verified though phantom measurements and analytical techniques by different groups. FIM is particularly sensitive and useful if the electrical impedance of a target object in the sensitive zone changes between two sets of measurements. This can be either due to a physical change in the target object, or, if its impedance has frequency dependence, through a change in the measurement frequency. In medical applications FIM is being developed for studying stomach and bladder emptying, localized lung function, detection of cervical and oral cancer, etc. FIM may have applications in industry to study physical mixtures of liquids and gases in pipelines and tanks, in geology to detect groundwater and other minerals, in oceanography, and in many other applications. Thus FIM appears to have a huge potential for application in different areas.
\end{abstract}

Keywords: Electrical Impedance technique, Focused Impedance Method, Volume conductor

\section{INTRODUCTION}

Passing a current through two terminals of a conductor and measuring the potential dropped, a measure of its electrical impedance is obtained. For a volume conductor, current flows in all directions from the electrodes and the sensitivity of different regions within the volume to the said measurement will depend upon the location of the region with respect to location of the electrodes. Again if a change in the conductivity occurs at any part of the volume conductor, this will also affect the measured impedance from which an assessment of the change can be made. However, if the volume conductor is a liquid (an electrolyte), contact potentials, polarisation and contact impedances (resistance and capacitance) arise at the electrode-liquid interface which may completely mask the impedance of the bulk material. Use of alternating currents (ac) in the measurement can eliminate the contact potentials to a large extent since they are basically dc and almost constant with time. Polarisation problems may also be eliminated through the use of ac. Contact impedances cannot be eliminated using the simple two electrode configuration. For this a slightly different technique known as Tetrapolar Impedance Method (TPIM) has been in use for a long time. Here four electrodes are used as shown in Fig. 1 in a 2 dimensional rendition of the method. Current $I$ is passed through two outer electrodes, while potential V developed across the other two are measured. Since the potential measuring devices have very high input impedances, current drawn through the two central potential electrodes are almost zero, rendering the potential dropped across

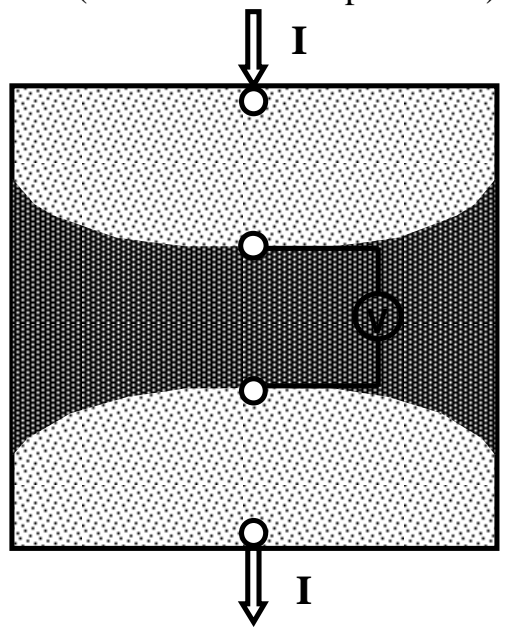

Fig.1. TPIM scheme and the wide sensitive zone between the equipotential lines of interest. 
the contact impedances negligible. Thus the potential measured is the true potential dropped across the bulk region between the two potential electrodes. The potential dropped across the contact impedances of the two current electrodes do not contribute to the potential measurement. In this method the equipotential surfaces passing through the two potential electrodes grossly mark the boundaries of the sensitive region, as shown by the central shaded region in Fig. 1. The potential developed across the potential electrodes equals the product of the current through the outer electrodes, and the impedance of the central shaded region as it appears to the two potential electrodes.

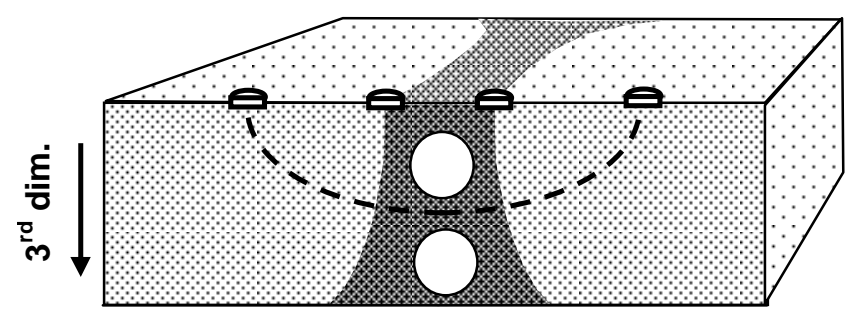

Fig.2. Extension of sensitive zone into the $3^{\text {rd }}$ dimension.

However, this is a too simplistic view of the situation and a proper analysis is needed to draw a detailed sensitivity distribution, which is often complex because of complex boundary conditions. An assessment using numerical methods for a semi-infinite 3D medium shows the salient features [1]. We use a simplistic physical view which gives a quick qualitative assessment. In this we state that the sensitivity at any point depends on the current density at that point and the perturbation in equipotential produced at the potential electrodes by a point object placed at that point. Therefore, for a medium with uniform conductivity, the sensitivity decreases from the centre outwards within the shaded region in Fig. 1.

An undesirable feature of a tetrapolar system is the presence of a region with negative sensitivity. This is because an object placed between the outer current electrodes and the adjacent potential electrodes will perturb the equipotential surfaces inversely to that done by an object placed in the central sensitive region. Therefore the effective impedance of the negative sensitive region will contribute to a reduction of the impedance desired to be measured. This is also demonstrated in the results obtained in [1]. Therefore while measuring the impedance of a large object extending beyond the central potential electrodes, one needs to interpret the results carefully.

However, if one is to measure the impedance of a localised object at a known location, which is embedded in a volume conductor of

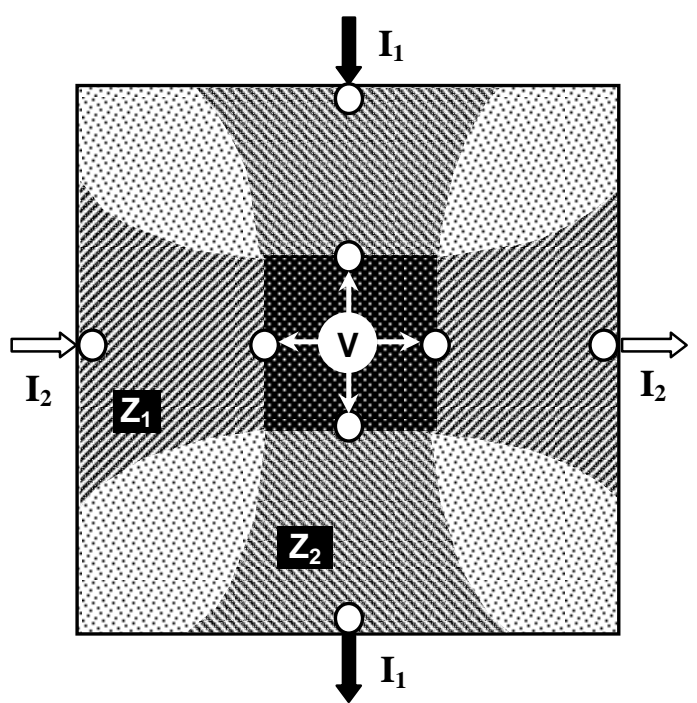

Fig.3. Basic concept of 8 electrode Focused Impedance Method (FIM). Central square zone has a dominant sensitivity, and is therefore, focused. uniform but different conductivity, then a practical method would be to ensure that the object lies within the central sensitive zone by proper placement of electrodes. This technique is particularly useful if the impedance of the target object changes between two sets of measurements while the background remains unchanged, even if it has regions with different conductivity. The above impedance change could be due 
to some time dependent activity, when measurement at two different times could indicate the change. Alternatively, if the impedance of the target object changes with frequency significantly but not that of the background, this change can be detected by performing the measurement at different frequencies of the measuring ac signal. Sometimes measurements at two frequencies only can give adequate information.

Although the discussions with respect to Fig. 1 relate more to a 2 dimensional situation, this can be extended to target objects in the $3^{\text {rd }}$ dimension using surface electrodes as shown in Fig. 2. The current gets distributed in the $3^{\text {rd }}$ dimension with the equi-current lines spreading out and current density decreasing with depth. The equipotential surfaces through the potential electrodes bend outwards with depth, encompassing more volume with increasing depth. Therefore within the sensitive zone, an object of a fixed size and shape placed near the surface will have more contribution to the measured impedance than when it is taken to increasing depths, which is akin to saying that the sensitivity decreases with depth.

Observing Fig.1, one point is clear that the sensitive zone is rather broad and if one wants to have localised information this is not the right system to work with. To overcome this difficulty Electrical Impedance Tomography (EIT, initially termed Applied Potential Tomography - APT) was developed [2] which uses a number of electrodes, typically 8,16 or 32 fixed transversely around a cylindrical volume in a particular plane, to image the 2 dimensional conductivity distribution in the said plane. However, since current flows in 3D, there is significant contribution to the image from points in the $3^{\text {rd }}$ dimension in a complex way [3], [4]. Thus the individual pixel values of the image do not give the right point sensitivity as expected. Presently attempts are being taken to obtain a 3D image by having more of the above set of electrodes in several planes along the length of the cylinder. 2D EIT itself is complex, and a 3D EIT is far more complex, and therefore it would be quite some time before practically usable 3D EIT systems are developed for regular use.

Focused Impedance Method (FIM), conceived by the first author [5], a simple technique of impedance measurement that can localize a zone of interest in a volume conductor eliminating effects of neighbouring regions. The basic concept of FIM involves two orthogonal and concentric TPIM measurements and summing the two measured impedances $\mathrm{Z}_{1}$ and $\mathrm{Z}_{2}$ as shown in Fig. 3. The central square zone contributes to the sum doubly than neighbouring regions, more so since it has a higher sensitivity even in a single TPIM measurement. Thus the central

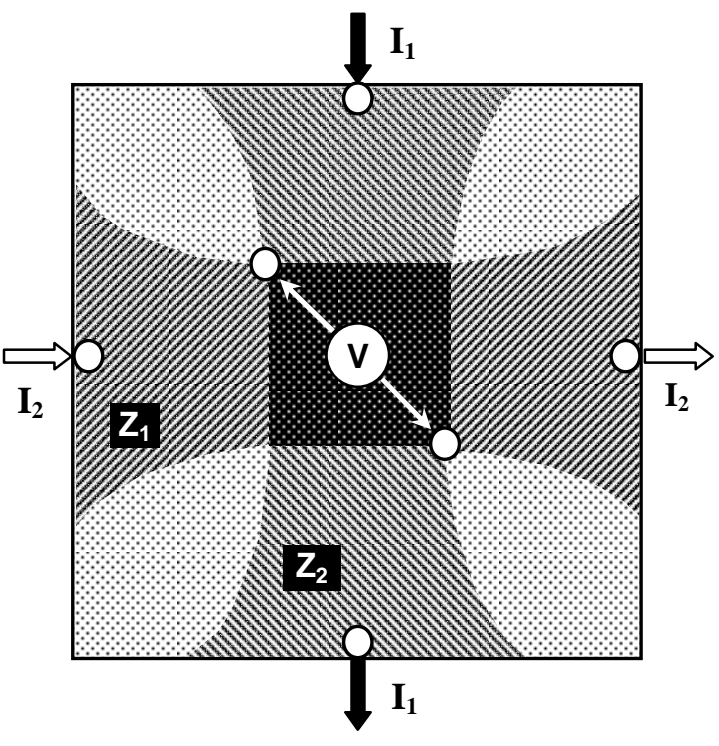

Fig.4. Basic concept of 6 electrode Focused Impedance Method.

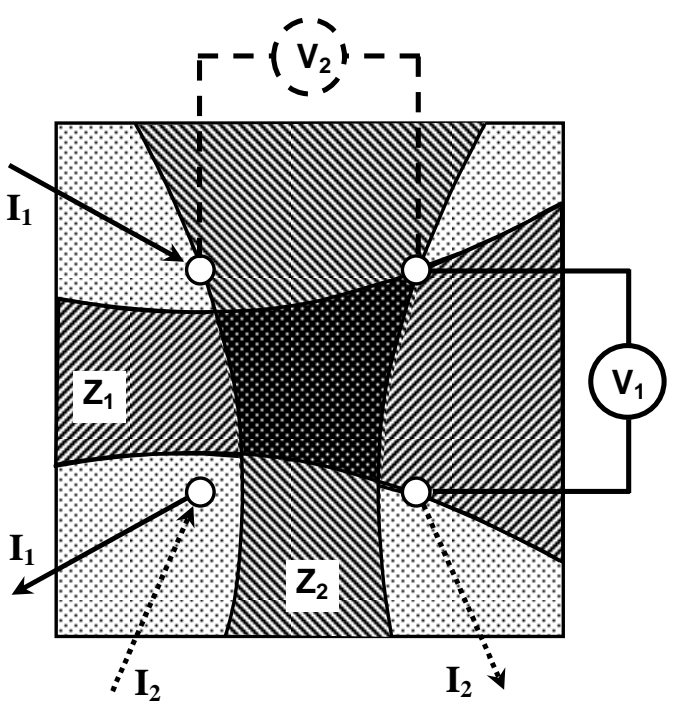

Fig.5. Basic concept of 4 electrode Focused Impedance Method 
region has a dominant contribution to the total summed value, i.e., in effect, the measurement focuses onto the central square region; hence the name, 'Focused Impedance'. This basic FIM requires 8 electrodes in addition to a common reference electrode. A modified version of above uses two potential electrodes placed at diagonal positions of the central focused region as shown in Fig. 4. Since each of these falls on the intersection of two equipotential lines, these two can replace the role of the four potential electrodes in Fig. 3. This version is called a 6 electrode FIM. Another version, 4 electrode FIM [6] was conceived as shown in Fig. 5. Here the four electrodes are configured differently, at corners of a square.

Current is passed through two adjacent electrodes and potential is measured across the opposite pair, and from this arrangement an impedance value is obtained. Then measurement is taken with the arrangement rotated through $90^{\circ}$. These have been indicated by $\mathrm{I}_{1}, \mathrm{Z}_{1}$ and $\mathrm{I}_{2}, \mathrm{Z}_{2}$ respectively. The sum of the two orthogonal impedance measurements, $Z_{1}+Z_{2}$ again gives a dominant sensitivity in the central region. This has been shown through regions bounded by appropriate equipotential lines in Fig. 5. The predictions of all the three FIM techniques have been borne out through 2D and 3D phantom measurements [5-7] and through numerical analysis [8].

\section{POTENTIAL APPLICATIONS}

This paper presents the potential applications of FIM in medicine, geology, industry, oceanography, and in microscopic studies. Since FIM was developed initially for applications in medicine, this aspect will be discussed in more detail. FIM is basically a bridge between age old TPIM which lacks in localisation, and the recent EIT giving an image which needs many electrodes, is sophisticated, and cannot give accurate images unless 3D EIT is developed. 2D EIT has seen some applications over the past two decades but it suffers from a contribution of objects in 3D, and a lack of accuracy in its pixel level image, as mentioned before. Therefore most studies using 2D EIT marked out a large region of interest in the image, and summed up all the pixel values. This ended up in a gross measurement of large localised volumes or organs within the body. In most cases changes in localised impedance over time were monitored which eliminated the non-changing background. In certain cases the target object, such as a tumour, has a frequency dependence which is different from that of the surrounding healthy tissue. This raises the possibility to detect such tumours and cancerous tissues using EIT spectroscopy using multi frequency measurements.

The rationale for developing FIM was that for such gross localised measurements, a sophisticated imaging method such as the EIT is not necessary, and FIM has been able to show success in this regard. Therefore, wherever 2D EIT with region of interest analysis has been applied [9], FIM can be applied with almost equal performance. Since FIM requires only a few electrodes and the instrumentation is simple, it has the potential of being applicable with a shorter development time.

\subsection{Biomedical Applications}

2.1.1 Gastric Emptying: Fig. 6 shows the result of a gastric (stomach) emtpying study after a drink of saline using FIM carried out by our extended group [5]. Skin surface electrodes, placed over the stomach region were used for this study. The change in FIM value clearly indicates the gradual emptying function, over a period of about 20

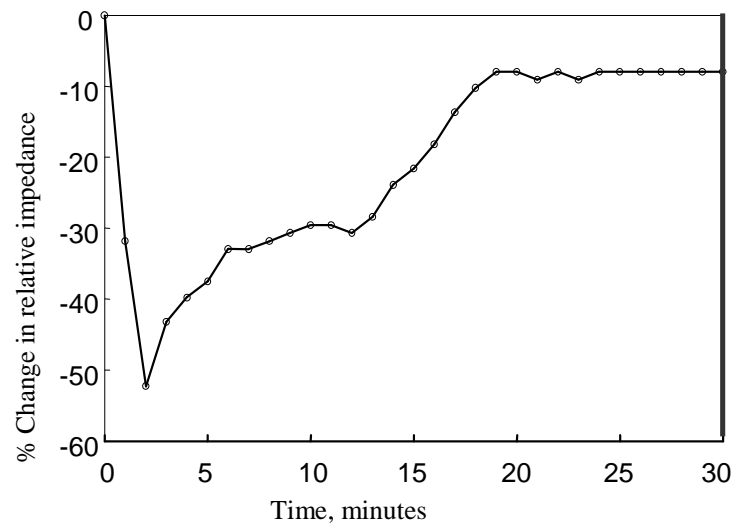

Fig.6. Gastric emptying using FIM after a drink of saline. 
minutes. Previous EIT studies [10] with a Region-of-Interest (ROI) marked around the stomach region gave exactly similar results. During this emptying procedure saline is expelled from the stomach to the duodenum in the lower right region and this was indicated in the EIT studies as well. During this time the impedance of the duodenum goes the opposite way. An earlier study by our extended group also showed that a TPIM measurement on the stomach did not give the expected behaviour, as it could not isolate the stomach from the duodenum. On the other hand FIM could do it successfully. This application of FIM shows that it can detect changes deep inside the body, in the $3^{\text {rd }}$ dimension with respect to the plane of the electrodes. The 3D sensitivity depends on the conductivity, volume and depth of the target volume [7]. Provided the conductivity and the depth are known, or are constant, FIM data can be related to the volume of saline in the stomach.

2.1.2 Gastric Acid Secretion: Low acid secretion has been linked to the proneness to diarrhoea, and to anaemia. However, no non-invasive technique exists to measure the volume of acid secreted. Existing techniques use intubation of stomach through the mouth and periodical withdrawal using a syringe; the procedure being grossly unpleasant to the subject. Research carried out by the authors' extended group at Dhaka using a 2D EIT system (made by the Sheffield group) with electrodes placed around the stomach region in a transverse plane showed a very good correlation between the intubation measurements and the ROI analysis of EIT data [11]. FIM can therefore be a good candidate for this application using surface electrodes placed over the stomach region.

\subsubsection{Lung Ventilation, perfusion and} mapping: FIM has particular advantages in studies of lung function. Dielectric properties of lungs tissue change as a function of air content and therefore impedance techniques can be used to study lungs ventilation. Our studies on lung ventilation demonstrated success of FIM [12]. Normal healthy subjects took a deep breath and then expired a little air at a time in steps and held the breath for a short while each time. During the breath holding period FIM data from the lower frontal right lobe of the lungs was taken. The volume of air expired was also measured simultaneously using a standard bellows type spirometer. Fig. 7 shows a typical data from one of the subjects and the very good correlation between the relative FIM values and the volume of air expired This shows the utility of FIM in measuring lung ventilation in localised regions, and from any direction, to identify zones with ventilation disorders. Moving the FIM electrodes over the thorax using a portable spring loaded electrode holder as shown in Fig. 8, mapping of the lungs is being carried out to establish standard parameters [13]. In future, the whole of the electronics may be compacted within this housing with digital numerical displays to make a portable stethoscope like instrument. FIM may be used to identify edema or abnormal masses

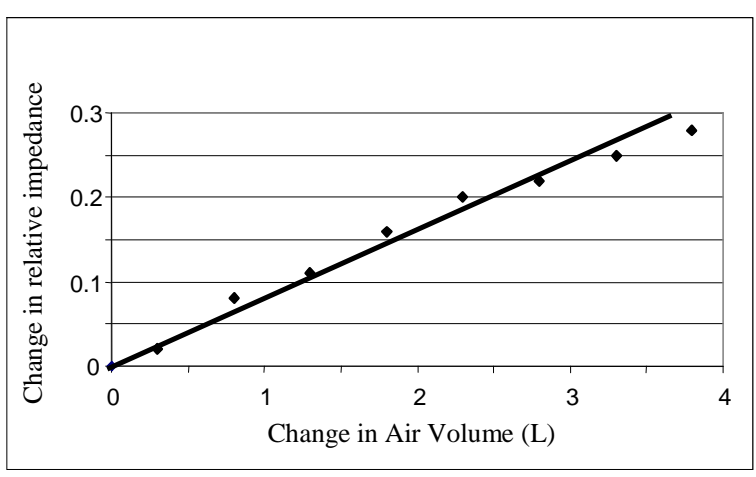

Fig. 7. Lung ventilation study using FIM showing linear relationship to change in air volume.

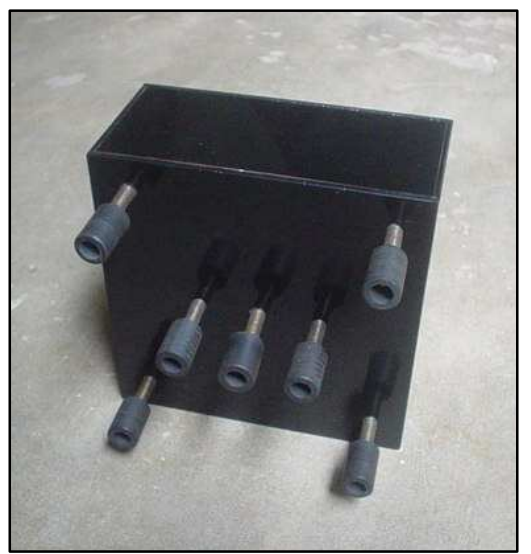

Fig. 8. Portable spring loaded electrode holder for lungs ventilation mapping using FIM. 
within the lungs, representing different diseases and disorders such as in TB or pneumonia, using FIM spectroscopy. FIM may also be useful in urgent monitoring of lungs ventilation of accident victims, since the equipment can be very small and portable. In artificial respiration of lungs, monitoring of lung compliance is extremely important and FIM may find useful application here. FIM can also be used to determine respiration rate simultaneously with other studies. With ECG gated instrumentation, FIM may also be used to determine lung perfusion by blood, and this in conjugation with lung ventilation study can provide very important diagnostic information on lung function.

2.1.4 Bladder Emptying: By placing electrodes at suitable points over the abdomen, bladder emptying during urination and the rate of urine flow may be monitored, which may help the diagnosis of prostate gland enlargement.

2.1.5 Cardio Vascular measurement: To measure the cardiac output, which is defined as the volume of blood pumped by the heart in one minute, TPIM and EIT techniques have been tried by different workers with limited success $[14,15]$. FIM has a potential here as it can directly look into a ventricle or atrium with appropriate placement of electrodes. TPIM and EIT (or Applied Potential Tomography) have been used with success in investigating vascular abnormalities [16,17]. FIM has therefore, a good potential in this area as well, as it can focus a small region anywhere on the body.

2.1.6 Tissue Temperature: The impedance of a body tissue depends on its temperature. So the temperature of a tissue may be sensed by impedance techniques and attempts have been made to use such techniques in measuring temperature change during hyperthermia treatment of tumours at deep sites [18]. Therefore, FIM with a localised target may be suitable for such measurement.

2.1.7 Diagnosis of cancer: The physical dimensions, internal structure, arrangement of the constituent cells, etc., determine the complex electrical impedance of a tissue. So the electrical impedance of normal tissue and that of cancerous tissue will be different. Reference [19] has shown that it is possible to distinguish between normal cells and abnormal precancerous cells in the cervix using a special arrangement of TPIM. Electrical impedance can also be used as a screening tool for skin cancer identification [20]. Therefore FIM has a potential in both these applications with improved zone localisation, and our extended group at Dhaka has already initiated work in this direction.

2.1.8 Abdominal Fat thickness: Localised abdominal fat is a risk indicator for diabetic and cardiovascular diseases. Abdominal fat content can be measured using electrical impedance methods as the resistivity of fat tissue is relatively high [21]. However, accurate determination of fat thickness from outside is not so straightforward. Our extended group at Dhaka has already initiated work to develop techniques to determine abdominal fat thickness using FIM [22].

\subsection{FIM in Geology}

Geophysical resistivity techniques are based on the response of the earth to the flow of electrical current giving a way to measure the electrical impedance of the subsurface material. Tetrapolar Electrical impedance methods have been in use for a long time in Geology for mining applications. Water saturation and connectivity of pore spaces can be measured by measuring the electrical impedance. This is because water has a low resistivity and electric current will follow the path of minimum resistance. Salinity of the underground water, porosity of rock and number of water filled fractures can also be detected using impedance technique. The compaction of soils or rock results increased impedance because compact air contains relatively less water. On the other hand air results in the opposite response compared to water when filling voids as air is highly resistive. It is also possible to detect the presence of mine (coal, oil, minerals etc) in the subsurface by measuring the impedance distribution [23]. Therefore FIM, having a localised sensitivity, may offer an improved procedure in all these applications. 


\subsection{Industrial applications}

Electrical impedance techniques have been tried in a number of industrial applications [24,25]. As suggested above, FIM may be applied in many of these applications. In determining the constituents in a mixture or slurry there is scope for application of FIM. It can also be used in the continuous monitoring of the flow of a liquid which may be mixed with air or other materials. Oil brought in tankers is sometimes mixed with water. There is a possibility to assess the amount of water using FIM as the petroleum is delivered through a pipeline.

\subsection{Oceanography}

In the study of the ocean floor and the study of the earth mantle, electrical impedance techniques are invaluable [26]. Again in the study of marine animals, in the study of the depth of oceans, in the detection of shoals of fish, electrical impedance techniques may have useful applications, and FIM may become an improved practical method.

\subsection{Microscopic measurements}

Electrical Impedance methods, particularly EIT has been taken up for various in-vitro studies on microscopic materials, life forms, and body tissues [27-29] using microelectrodes. FIM may perform equally in similar applications as it has less number of electrodes to work with.

\section{CONCLUSION}

It appears that electrical impedance techniques have a large potential in different fields of measurements. However, these could not make an expected impact so far. This is possibly due to the wide area or volume of sensitivity for TPIM systems on one hand, and in the inaccuracy of pixel level images in EIT together with a complexity of instrumentation. Therefore, FIM being a bridge between the two, appears to bring about the impact sooner if pursued in all these applications. Having less number of electrodes to deal with and simple instrumentation, and offering a reasonable target zone of measurement that can be manipulated through electrode spacing, FIM appears to be a technique with great promise.

\section{REFERENCES}

1. B. H. Brown., A. J. Wilson and P. Bertemes Filho, "Bipolar and tetrapolar transfer impedance measurements from volume conductor," Electronics Letters, vol. 36, pp. 2060-2062, 2000.

2. D. C. Barber, B. H. Brown and I. L. Freeston, "Imaging spatial distributions of resistivity using applied potential tomography," Electron. Lett., Vol. 19, pp. 933-5, 1983.

3. K. S. Rabbani, H. Kabir, "Studies on the effect of the third dimension on a two dimensional Electrical Impedance Tomography system," Clin. Phys. Physiol. Meas, vol. 12, pp. 393-402, 1991.

4. K. S. Rabbani, M. Hassan, A. Kiber, "3D object localisation using EIT measurement at two levels," Physiol. Meas., Vol. 17, pp. 189-99, 1996.

5. K. S. Rabbani, M. Sarker, M. H. R. Akond and T. Akter, "Focused Impedance measurement (FIM) - A new technique with improved zone localization," Ann. New York Acad. Sci., vol. 873, pp.408-420, 1999.

6. K. S. Rabbani and M. A. S. Karal, "A new four-electrode Focused Impedance Measurement (FIM) system for physiological study," Ann. Biomed. Engg. (Springer), vol 36, pp.1072-1077, 2008.

7. H. M. Iquebal and K. S. Rabbani, "3D sensitivity of 6-electrode Focused Impedance Method (FIM)," J. Phys.: Conf. Ser. (IOP Publishing), vol. 224, No.1, 2010. [Online]. Available: http://iopscience.iop.org/17426596/224/1/012156/pdf

8. N. Islam, K. S. Rabbani, A. Wilson, "The sensitivity of focused electrical impedance measurements," Physiol. Meas. Vol. 31, pp. S97-S109, 2010 [Online]. Available: http://iopscience.iop.org/0967-3334/31/8/S08/pdf

9. BH Brown, DC Barber and AD Seagar Applied potential tomography: possible clinical applications Clin. Phys. Physiol. Meas., vol. 6, pp. 109-21, 1985 
10. R. Avill, Y. F. Mangnall, N. C. Bird, B. H. Brown, D. C. Barber, A. D. Seagar, A. G. Johnson and N. W. Read, "Applied potential tomography: A new non invasive technique for measuring gastric emptying," Gastroenterology vol. 92, pp. 1019-26, 1987.

11. S. A. Sarker, D. Mahalanabis, P. K. Bardhan, N. H. Alam, K. S. Rabbani, A. Kiber, M. Hassan, S. Islam, G. J. Fuchs and K. Gyr, "Noninvasive Assessment of Gastric Acid Secretion in Man (Application of Electrical Impedance Tomography (EIT))," Digestive Diseases and Sciences (Springer Netherlands), vol. 42, pp.18041809, 1997.

12. M. A. Kadir, T. N. Baig. K S Rabbani, "Application of 6-electrode Focused Impedance Method (FIM) to study lungs ventilation," in Proc. EIT2009, UK, [Online]. Available: http://www.maths.manchester.ac.uk/eit2009/abstracts/kadir.pdf

13. M A Kadir, H Ferdous, T N Baig and K S Rabbani, Ventilation mapping of chest using Focused Impedance Method (FIM), J. Phys.: Conf. Ser. (IOP Publishing), vol. 224, No.1, 2010. [Online]. Available: http://iopscience.iop.org/1742-6596/224/1/012031

14. D. P. Bernstein, "Continuous noninvasive real-time monitoring of stroke volume and cardiac output by thoracic electrical bioimpedance," Critical Care Medicine, vol. 14, pp. 898-901, October 1986.

15. H. Luepschen, T. Muders, R. Pikkemaat, S. Leonhardt, T. Meier, H. Wrigge, G. Hedenstierna, C. Putensen, "Noninvasive Stroke Volume Measurement With Electrical Impedance Tomography At Grossly Changing Lung Conditions," Am. J. Respir. Crit. Care Med., vol. 181, p. A4581, May, 2010

16. W. S. Coxe, H. B. Shumacker, Jr., L. W. Freeman, "Impedance plethysmography in study of peripheral circulation," AMA Arch Surg. Vol. 65, pp. 611-620, 1952.

17. Ahmed, "Study of peripheral circulation in non-pregnant, pregnant and pre-eclamptic women using applied potential tomography," Australian and New Zealand J. of Obstet. and Gynae., vol. 44, pp. 322-327, 2004

18. H. M. Amasha., A. P Anderson., J. Conway, D. C. Barber, "Quantitative assessment of impedance tomography for temperature measurement in microwave hyperthermia," Clin. Phys. Physiol. Meas., vol. 9, Suppl. A, pp. 49$54,1988$.

19. H. Brown, J. A. Tidy, K. Boston, A. D. Blackett, R. H. Smallwood, F. Sharp, "Relation between tissue structure and imposed electrical current flow in cervical neoplasia," The Lancet, vol. 355, pp. 892-895, 2000.

20. Peter Aberg, Ingrid Nicander, Johan Hansson, Paul Geladi, Ulf Holmgren, and Stig Ollmar, Skin Cancer Identification Using Multifrequency Electrical Impedance- A Potential Screening Tool, IEE Transactions on Biomedical Engineering, VOL.51, NO.12, December 2004

21. H Scharfetter, T Schlager, R Stollberger, R Felsberger, H Hutten and H Hinghofer-Szalkay, Assessing abdominal fatness with local Bioimpedance analysis: Basics and experimental findings. International Journal of obesity, vol. 25, pp. 502-511, 2001.

22. S Haowlader, T N Baig, K S Rabbani, Abdominal fat thickness measurement using Focused Impedance Method (FIM) - phantom study, J. Phys.: Conf. Ser. (IOP Publishing), Vol. 224, No., 2010 [Online]. Available: http://iopscience.iop.org/1742-6596/224/1/012061/pdf

23. R. A. Eso, D. W. Oldenburg, M. Maxwell, "Application of 3D electrical resistivity imaging in an underground potash mine," in Proc. SEG Annual Meeting / New Orleans 2006, Extended Abstract 25, pp. 629-632.

24. T. York, "Status of electrical tomography in industrial applications," J. Electron. Imaging, Vol. 10, pp. 608, 2001.

25. K. A. Shollenberger, J. R. Torczynski, T. J. O’Hern, D. R. Adkins, S. L. Ceccio, D. L. George, “Comparison of Gamma-Densitometry Tomography and Electrical Impedance Tomography for Determining Material Distribution in Liquid-Solid Flows," in Cavitation and Multiphase Flow Forum, J. Katz \& K. J. Farrell, eds., FEDSM97-3690, 1997.

26. Y. Takashi, M. Laumonier, E. McIsaac, T. Katsura, "Electrical conductivity of basaltic and carbonatite meltbearing peridotites at high pressures: Implications for melt distribution and melt fraction in the upper mantle," Earth Planet. Sci. Lett., vol. 295, pp. 593-602, 2010.

27. H. Griffiths, M. G. Tucker, J. Sage, W. G. Herrenden-Harker, "An electrical impedance tomography microscope," Physiol. Meas., vol. 17, pp. A15, 1996.

28. BF De Blasio, M Laane, T Walmann, I Giaever, "Combining optical and electrical impedance techniques for quantitative measurement of confluence in MDCK-I cell cultures," Biotechniques, vol. 36, pp. 650-4, Apr. 2004.

29. H. Liu, A. R. Hawkins, S. M. Schultz, T. E. Oliphan, "Microscopic impedance imaging of small tissues," in Proc. 3rd IEEE International Symposium on Biomedical Imaging, Arlington, USA, 2006, pp. 1084 - 1087. 